ARAÚJO, A.M.S. Plasma Seminal (Revisão). PUBVet, Londrina, V. 8, N. 18, Ed. 267, Art. 1775, Setembro, 2014.

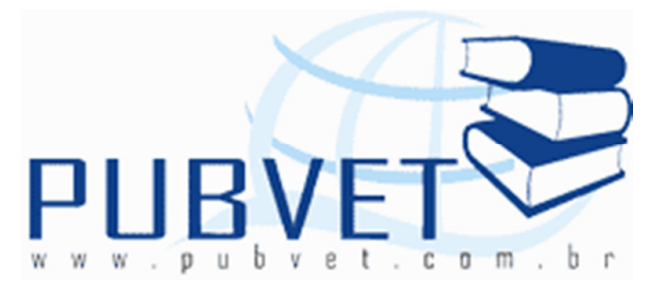

PUBVET, Publicações em Medicina Veterinária e Zootecnia.

\title{
Plasma Seminal (Revisão)
}

\section{Amanda Melo Sant'Anna Araújo}

Médica Veterinária formada na Universidade Federal Rural do Rio de Janeiro, Especialista em Acupuntura pelo Instituto de Acupuntura do Rio de Janeiro e Mestre em Ciência Animal pela Universidade Estadual do Norte Fluminense.

\section{Resumo}

O plasma seminal serve como veículo para os espermatozóides ejaculados, consistindo numa mistura de secreções do testículo e glândulas acessórias masculinas. Possui um significado fisiológico importantíssimo como carreador dos gametas masculinos até o trato genital feminino, dando condições para a viabilização do processo de fertilização. Já foi constatado que o conteúdo do plasma seminal pode influenciar na fertilidade dos machos. Estudos indicam que a habilidade do espermatozóide para se ligar à heparina e a outros glicosaminoglicanos está correlacionada com a qualidade do sêmen e fertilidade. As proteínas com sítio de ligação para heparina no plasma seminal influenciam na fertilidade. Plasma seminal parece protege os espermatozóides de serem fagocitados.

Palavras-chave: espermatozóide, testículo, fertilidade, sêmen 
ARAÚjO, A.M.S. Plasma Seminal (Revisão). PUBVet, Londrina, V. 8, N. 18, Ed. 267, Art. 1775, Setembro, 2014.

\title{
Seminal Plasma (Review)
}

\begin{abstract}
Seminal plasma serves as a vehicle for ejaculated spermatozoa consisting of a mixture of secretions from the male accessory glands and testis. Has an important physiological significance as a carrier of the male gametes to the female genital tract, giving conditions for enabling the fertilization process. It had been found that the content of seminal plasma may influence male fertility. Studies indicate that the ability of sperm to bind to heparin and other glycosaminoglycans is correlated with semen quality and fertility. The proteins with binding site for heparin in seminal plasma influence on fertility. Seminal plasma protects spermatozoa seems to be phagocytosed.
\end{abstract}

Keywords: sperm, testis, fertility, semen

\section{Introdução}

A avaliação reprodutiva do macho cursa com a avaliação de diversos parâmetros, onde a fertilidade é determinada após exames clínicos, exame específico do aparelho reprodutivo e avaliação da qualidade seminal. Mas estas avaliações muitas vezes podem ser insuficientes para predizer o potencial fecundante do sêmen.

Durante o processo de congelamento os espermatozóides sofrem vários danos, dendre eles destacam-se: alterações de membrana, capacitação prematura, alterações no DNA e o estresse oxidativo.

O plasma seminal é constituído de uma mistura de secreções dos testículos, epidídimos e glândulas acessórias que serve como meio para os espermatozóides ejaculados (HENALUT et al., 1995). Esse fluído complexo contém diversos polipeptídeos, que exercem um importante papel na capacidade fertilizante dos espermatozóides ( MARQUES et al., 2000).

É sabido que o plasma seminal tem muitas funções fisiológicas e imunológicas nas diferentes espécies (LAZAREVIC et al, 1995;. THERIEN et al, 1997;. ALGHAMDI et al, 2004;. ALGHAMDI e FOSTER, 2005; SHARKEY et al, 
ARAújo, A.M.S. Plasma Seminal (Revisão). PUBVet, Londrina, V. 8, N. 18, Ed. 267, Art. 1775, Setembro, 2014.

2007).. Como as células espermíaticas precisam se submeter a capacitação, serem transportadas ao site de fertilização, e evitar fagocitose prematura, fatores diferentes no plasma seminal coordenam essas atividades para otimizar as chances de espermatozóides para fertilizar os oócitos. Durante a ejaculação, os espermatozóides e fluido epididimário são misturados com secreções das glândulas sexuais acessórias (ASGs), que não parecem para "ejetar" o seu conteúdo simultaneamente (THERIEN et al, 1995; . NAUC E MANJUNATH, 2000; BJORNDAHL E KVIST, 2003; WEBER E WOODS, 1993).

Porém, estudos sobre as proteínas do plasma seminal e da membrana espermática ainda precisam definir os tipos de proteínas e os mecanismos de ação que afetam a viabilidade desses gametas (ASADPOUR et al., 2007).

WELTER (2006) concluiu que existe uma grande variedade de proteínas no plasma seminal, e essas podem estar correlacionadas com as alterações de cromatina, a morfologia espermática e a morfometria de cabeça, revelando o poder dessas proteínas atuarem como marcadores bioquímicos.

\section{Revisão de literatura}

\subsection{Função do plasma seminal}

O plasma seminal serve como veículo para os espermatozóides ejaculados, consistindo numa mistura de secreções do testículo e glândulas acessórias masculinas. Possui um significado fisiológico importantíssimo como carreador dos gametas masculinos até o trato genital feminino, dando condições para a viabilização do processo de fertilização RONCOLETTA et al (1999).

Os espermatozódes e o plasma seminal possuem substâncias enzimáticas e não-enzimáticas que servem como mecanismo de defesa contra a ROS. Além dassas enzimas, o plasma seminal possuem outros componestes que agem como antoxidantes, entre eles vitaminas E e C, urato, albumina, taurina e hipotaurina (ALMEIDA e BALL, 2005). 
ARAÚJO, A.M.S. Plasma Seminal (Revisão). PUBVet, Londrina, V. 8, N. 18, Ed. 267, Art. 1775, Setembro, 2014.

Tem sido demonstrado que o plasma seminal atua na manutenção da motilidade e viabilidade espermática (MAXWELL et al, 1 996), além de aumentar a resistência dos espermatozóides ao choque térmico (MORTIMER \& MAXWELL, 2004).

O plasma seminal proporciona boas condições para a manutenção da motilidade, da sobrevivência e do transporte espermático, tanto no sistema reprodutor do macho, quanto da fêmea (TÖPFER-PETERSEN et al., 2004). Entretanto, estudos sobre as proteínas do plasma seminal e da membrana espermática ainda precisam definir os tipos de proteínas e os mecanismos de ação que afetam a viabilidade desses gametas (ASADPOUR et al., 2007).

A composição molecular do plasma seminal possui características inerentes a cada espécie, podendo diferir entre os tipos e a atuação das proteínas espermáticas. O plasma seminal dos Bovídeos possui a família das proteínas designadas de BSP-A1/-A2 e BSP-A3 (15-17kDa), BSP-30 (2830kDa) (MANJUNATH, 1984), e FMP, relacionadas à motilidade progressiva (37,5kDa). Em eqüinos, as proteínas HSP-1 (22-25kDa), HSP-2 (25kDa, pI 6,5-6,9), HSP-7 (14kDa) e HSP-12 kDa pertencem à família das espermadesinas, e a SP-1 possui correlação positiva com a fertilidade (TÖPFER-PETERSEN et al., 2004).

O plasma seminal contém proteínas que participam de processos relacionados à proteção dos espermatozoides (KRAUS et al., 2005) durante o trânsito epididimário e o armazenamento na cauda deste órgão (HINTON et al., 1995), por ocasião da ejaculação e no trato reprodutor da fêmea. Apesar de o processo de formação de espécies reativas de oxigênio (ROS) desempenhar função importante na fisiologia do espermatozoide (MACLEOD, 1943), um desequilíbrio nessa formação pode levar ao estresse oxidativo com consequentes distúrbios funcionais do espermatozoide, por meio de mecanismos como peroxidação lipídica (AITKEN et al., 1993), redução da atividade de enzimas reguladoras do influxo de cálcio na membrana (OHTA et al., 1989) e perda de ATP (DE LAMIRANDE E GAGNON, 1992). Para combater a 
ARAÚjO, A.M.S. Plasma Seminal (Revisão). PUBVet, Londrina, V. 8, N. 18, Ed. 267, Art. 1775, Setembro, 2014.

ação deletéria das ROS sobre os espermatozoides, o epidídimo secreta enzimas antioxidantes (Hinton et al., 1996),

Já foi constatado que o conteúdo do plasma seminal pode influenciar na fertilidade dos machos. Estudos indicam que a habilidade do espermatozóide para se ligar à heparina e a outros glicosaminoglicanos está correlacionada com a qualidade do sêmen e fertilidade. As proteínas com sítio de ligação para heparina no plasma seminal influenciam na fertilidade. Estes sítios de ligação têm estrutura semelhante a proteínas (GAGS) do líquido folicular ovariano, que são responsáveis pela estimulação das reações acrossômicas no espermatozóide bovino, de coelho e porco, agindo provavelmente durante a capacitação do espermatozóide (MILLER et al. 1990).

Em éguas é normal ocorrer endometrite que é uma inflamação fisiológica transitória que serve para limpar o excesso de espermatozóides mortos e outros contaminantes do útero após o acasalamento (TROEDSSON, 1999). A inflamação é causada por espermatozóides e se caracterizada por um rápido influxo de neutrófilos polimorfonucleares para o lúmen uterino dentro uma hora após a inseminação artificial (IA), e dura até 36 h em éguas normal (KOTILAINEN et al. 1994, KATILA 1995, TROEDSSON 1999, TROEDSSON et al. 2000, 2001).

A hipótese de Alghamdi et al. (2004) é que o plasma seminal suprime a fagocitose de espermatozóides, inibindo neutrófilos polimorfosnucleares vinculados a espermatozódes. E confirmaram que uma substância proteica de plasma seminal de eqüinos reduz a ligação de inibindo neutrófilos polimorfosnucleares vinculados aespermatozóides in vitro, e que o plasma seminal melhora a fertilidade dos espermatozóides frescos inseminadas em uteros inflamados. Além disso essa substância pode ser precipitada a partir do plasma seminal, aquecida até $56^{\circ} \mathrm{C}$ por $3 \mathrm{~h}$ e após ser congelado sem perder a suas propriedades biológicas.

Plasma seminal parece proteger os espermatozóides de serem fagocitadas por PMNs em vitro (TROEDSSON et al. 2000). Os dados de ALGHAMDI et al. (2004) sugeriram que esta proteção é de pelo menos, em 
ARAÚjO, A.M.S. Plasma Seminal (Revisão). PUBVet, Londrina, V. 8, N. 18, Ed. 267, Art. 1775, Setembro, 2014.

parte, mediado pela redução de espermatozóides vincilados a neutrófilos polimorfosnucleados. A função de proteção de plasma seminal podem ser específicos para determinadas sub populações de espermatozóides, e/ou ocorrer apenas durante uma janela temporária de tempo, permitindo que as células de esperma para alcançar o oviduto, a fim para fertilizar um óvulo.

\subsection{Criopreservação}

A congelabilidade do sêmen é um fator bastante importante para a melhoria do material biológico para a realização e sucesso da técnica de Inseminação Artificial e conseqüentemente para o Melhoramento Genético Animal conforme RONCOLETTA et al. (1999). Alguns fatores impedem que o sêmen eqüino congelado possa ser utilizado em larga escala, dentre eles, a variação individual frente a criopreservação e o baixo rendimento de doses por ejaculado (MORRIS et al., 2003).

Proteínas do plasma seminal bovino (BSP) foram identificadas como tendo a propriedade de ligação à superfície do espermatozóide, diminuindo sua quantidade após a criopreservação do sêmen, sugerindo alguma relação com a congelabilidade do sêmen (NAUC E MANJUNATH, 2000). Entretanto, é desconhecida a influência destas proteínas sobre o processo de criopreservação do sêmen. Por outro lado, a presença de um fator polipeptídico de $61,8 \mathrm{kDa}$ no plasma seminal de touros da raça GIR foi identificado como associado à congelabilidade do sêmen desta raça (RONCOLETTA et al., 1999).

A grande variabilidade na constituição do plasma seminal, principalmente em seu conteúdo protéico, pode explicar as diferenças individuais na funcionalidade de espermatozóides, resultando em diferentes respostas ao congelamento e fertilidade após a inseminação artificial (MAXWELL \& JOHNSON, 1 999).

Há evidências consideráveis da influência do plasma seminal sobre a resistência de espermatozóides ao choque térmico e sua capacidade de sobreviver após o descongelamento (PURSEL \& JONHSON, 1975). Efeitos benéficos da utilização do plasma seminal na criopreservação de 
ARAÚJO, A.M.S. Plasma Seminal (Revisão). PUBVet, Londrina, V. 8, N. 18, Ed. 267, Art. 1775, Setembro, 2014.

espermatozóides de equinos (AURICH et al., 1996; KATILA et al., 2002; BARRETO et al., 2008), bovinos (RONCOLETTA et al., 2002), humanos (BEN et al., 1997), suínos (METZ et al., 1990) e ovinos (GHAOUI et al., 2007) têm sido descritos. Esses efeitos são devidos, possivelmente, à interação de proteínas do plasma seminal com os espermatozóides, o que pode influenciar sua capacidade fertilizante e a proteção da membrana espermática ao choque térmico (BARRIOS et al., 2000).

AMANN (1995) discorreu que para a criopreservação é necessário um exame rigoroso na seleção dos ejaculados, a qual serve como suporte para apurar o potencial de fertilidade do animal. Dentro do conceito fertilidade, o fator congelabilidade é muito pouco mencionado e discutido, sendo poucos os autores que o mencionaram, associaram-no com constituintes do plasma seminal, como proteínas totais, sólidos totais, frutose, GOT e GTP segundo DHAMI e KODAGALI (1987); e sugeriram os alelos HLA-DPB como marcadores moleculares específicos para parâmetros de descongelação do sêmen (PERROTT et al., 1992).

\section{Considerações finais}

É evidente a importância do plasma seminal na fertilidade do macho, ressaltando a proteção a qual promove nas células espermáticas em especial às membranas destas células que precisam estar íntegras para percorrer todo o trato reprodutivo da fêmea e apenas no momento adequado devem devem se desestabilizar.

\section{Bibliográficas}

AITKEN RJ, HARKISS D, BUCKINGHAM D. Relationship between ironcatalysed lipid peroxidation potential and human sperm function. Journal of Reproduction and Fertility, v.98, p.257$265,1993$.

ALGHAMDI, A.S., FOSTER, D.N., Seminal DNase frees spermatozoa entangled in neutrophil extracellular traps. Biology of Reproduction 73, 1174-1181. 2005. 
ALGHAMDI1, A.S.; FOSTER, D.N.; TROEDSSON, M.H.T., Equine seminal plasma reduces sperm binding to polymorphonuclear neutrophils (PMNs) and improves the fertility of fresh semen inseminated into inflamed uteri. Reprodution. 127 593-600. 2004.

ALGHAMDI, A.S., FOSTER, D.N., TROEDSSON, M.H., Equine seminal plasma reduces sperm binding to polymorphonuclear neutrophils (PMNs) and improves the fertility of fresh semen inseminated into inflamed uteri. Reproduction 127, 593-600. 2004.

AMANN, R.P. Evaluation of sperm quality: can we pick the winners? In: CONGRESSO BRASILEIRO DE REPRODUÇÃO ANIMAL, 11. Belo Horizonte : CBRA, 1995. Anais. Belo Horizonte : CBRA. p.206-12. 1995.

AURICH, J.E., KÜHNE, A., HOPPE, H., AURICH, C. Seminal plasma affects membrane integrity and motility of equine spermatozoa after cryopreservation.

Theriogenology, v. 46, p. $791-797,1996$.

ASADPOUR, S.M. et al. SDS-polyacrylamide gel electrophoresis of buffalo bulls seminal plasma proteins and their relation with semen freezability. Animal Reproduction Science, v.102, p.308313, 2007.

BARRIOS, B., PÉREZ-PÉ, R., GALlEGo, M., TATO, A., OSADA, J., MUIÑO-BLANCO, T., CEBRIÁNPÉREZ, J.A. Seminal plasma proteins revert the coldshock

damage on ram sperm membrane. Biology of Reproduction, v.63, p. 1531 -1 537, 2000.

BARRETO, M.A.P.; SILVA, J.F.S.; FAGUNDES, B.; CAIADO, J.R.C.; SOUZA, G. V.; SHIMOYA, A. Efeito de proteínas do plasma seminal eqüino com massa superior a $10 \mathrm{kDa}$ concentradas 10 vezes sobre a congelabilidade do sêmen. Revista Brasileira de

Zootecnia, v. 37, p. 211 5-211 9, 2008.

BEN, W.X., FU, M.T., MAO, L.K., MING, Z.W., XIONG, W.W. effect of various concentrations of native seminal plasma in cryoprotectant on viability of human sperm. Archives of Andrology, v.39, p.211 -216, 1997.

BJORNDAHI, L., KVIST, U., 2003. Sequence of ejaculation affects the spermatozoon as a carrier and its message. Reproduction Biomedicine On line 7, 440-448.

DHAMI, A.J.; KODAGALI, S.B. Correlation between biochemical and enzymatic constituents of semen of Surti buffalo bulls. Indian Journal of Animal Sciences, v.57, n.12, p.1283-6, 1987.

GHAOUI, R. E., THOMSON, P.C., LEAHY, T., SALAMON, G., MAXWELL, W.M.C. Autologous whole ram seminal plasma and its vesicle-free fraction improve motility characteristics and membrane status but not in vivo fertility of frozen-thawed ram spermatozoa. Reproduction in Domestic Animals, v.42, p.541-549, 2007.

HENALUT, M.A.; KILLIAN, G.J.; J.F.KAVANAAUGH, J.F.; GRIL, L.C. Effect of accessory sex gland fluid from bulls of different fertilities on the ability of cauda epididymal sperm to penetrate zona-free ovine oocytes. Biology of Reproduction, v.52, p. 390-397, 1995.

HINTON BT, PALLADINO MA, RUDOLPH D, LABUS JC. The epididymis as protector of maturing spermatozoa. Reproduction and Fertility Develooment, v.7, p.731-745, 1995.

KATILA T Onset and duration of uterine inflammatory response of mares after insemination with fresh semen. Biology of Reproductive Monograph Series, 1 515-517, 1995.

KATILA, T., ANDERSON, M., REILAS, T., KOSKINEN, E. Post-thaw motility and viability of fractionated and frozen stallion ejaculates. Theriogenology, v.58, p.241 -244, 2002. 
KOTILAINEN, T.; HUHTINEN, M.; KATILA, T. Sperm-induced leukocytosis in the equine uterus. Theriogenology 41 629-636, 1994.

KRAUS M, TICHÁ M, ZELEZNÁ B. Characterization of human seminal plasma proteins homologous to boar AQN spermadhesins. Journal of Reproduction Immunology, v.65, p.33-46, 2005.

LAMIRANDE, E.; GAGNON, C. Reactive oxygen species and human spermatozoa. I. Effects on the motility on intact spermatozoa and sperm axonemes. Journal Andrology, v.13, p.368-378, 1992.

LAZAREVIC, M.; SKIBINSKI, G.; Kelly, R.W.; James, K., 1995. Immunomodulatory effects of extracellular secretory vesicles isolated from bovine semen. Vet. Immunol. Immunopathol. 44, 237-250.

MACLEOD, J. The role of oxygen in the metabolism and motility of the human spermatozoa. American Journal Physioogyl, v.138, p.512-518, 1943.

MANJUNATH, P. Gonadotropin release stimulatory and inhibitory proteins in Bull seminal plasma. In: SAIRAM, M.R.; ATKINSON, L.E. (Eds.) Gonadal proteins and peptides and their biological significance. Singapore: World Scientific, p.49-61, 1984.

MAXWELL, W.M.;WELCH, G.R.; JOHNSON, L.A. Viability and membrane integrity of spermatozoa after dilution and flow cytometric sorting in the presence or absence of seminal plasma. Reproduction, Fertility and Development, v.8, p.11 65-11 78, 1996.

MAXWELL, W.M.C.; JOHNSON, L.A. Physiology of spermatozoa at high dilution rates: the influence of seminal plasma. Theriogenology, v.52, p.1 353-1 362, 1999.

MILLER, D.J.; WINER, M.A.; AX, R.L. Heparin-binding proteins from seminal plasma bind to bovine spermatozoa and modulate capacitation by heparin. Biology of Reproduction, v.42, p.899-915, 1990.

MORRIS, L.H.A., TIPLADY, C., ALLEN, W.R. Pregnancy rates in mares after a single

fixed time hysteroscopic insemination of low numbers of frozen-thawed spermatozoa onto the uterotubal junction. Equine Veterinary Journal, v. 35, n. 2, p. 197-201, 2003.

MORTIMER, S.T., MAXWELL, W.M.C. Effect of medium on the kinematics of frozen-thawed ram spermatozoa. Reproduction, v.1 27, p.285-291, 2004.

NAUC, V.,; MANJUNATH, P. Radioimmunoassays for bull seminal plasma proteins (BSP-A1/-A2, BSP-A3, and BSP-30- Kilodaltons), and their quantification in seminal plasma and sperm. Biology Reprouction. 63, 1058-1066, 2000.

PERROTT, W.; SU, B.C.; CHAN, P.J.; KALUGDAN, D.R.; TREDWAY, D.R. Identification of sperm cryopreservation - thawing parameters based on HLA-DPB allelic sequence variation determined through the polymerase chain reaction (PCR). In: MEETING SOCIETY OF AMERICAN FERTILITY, 18. Abstracts, 1992. p.59-133, 1992

PURSEL, V.G., JONHSON, L.A. Freezing of boar spermatozoa: fertilizing capacity with concentrated semen and a new thawing procedure. Journal of Animal Science, v.40: p.99-1 02, 1975.

RONCOLETTA, M.; MORANI. E.S.C.; FRANCESCHINI, P.H. $14 \mathrm{kDa}$ seminal plasma protein identification and its relation with bull semen freezability. Theriogenology, v.57, p.479, 2002. 
RONCOLETTA, M.; MORANI. E.S.C.; FRANCESCHINI, P.H.; LIMA, V. F. M.H.; RODRIGUES, L. H.; OLIVEIRA, M. A.; SILVA, C. Perfil em SDS-PAGE das proteínas do plasma seminal e sua relação com a congelabilidade do sêmen de touros doadores da raça gir Brazilian Journal of Veterinary Research and Animal Science v.36 n.2 São Paulo 1999.

SHARKEY, D.J.; MACPHERSON, A.M.; TREMELLEN, K.P.; ROBERTSON, S.A. Seminal plasma differentially regulates inflammatory cytokine gene expression in human cervical and vaginal epithelial cells. Molecular Human Reproduction. 13, 491-501, 2007.

THERIEN, I., BLEAU, G., MANJUNATH, P., Phosphatidylcholine-binding proteins of bovine seminal plasmamodulate capacitation of spermatozoa by heparin. Biology Reproduction, 52, 1372-1379, 1995.

THERIEN, I., SOUBEYRAND, S., MANJUNATH, P. Major proteins of bovine seminal plasma modulate sperm capacitation by high-density lipoprotein. Biology Reproduction 57, 1080$1088,1997$.

TÖPFER-PETERSEN, E. The role of stallion seminal proteins in fertilization. Animal Reproduction Science, v.89, n.1-4, p.159-170, 2004.

TROEDSSON M.H.T. Uterine clearance and resistance to persistent endometritis in the mare. Theriogenology 52 461-471, 1999.

TROEDSSON M.H.T., LEE C-S, FRANKLIN R.D.; CRABO B.G. The role of seminal plasma in post-breeding uterine inflammation. Journal of Reproduction and Fertility Supplement 56 341349,2000

TROEDSSON MHT, LOSET K, ALGHAMDI AM, DAHMS B \& CRABO BG Interaction between equine semen and the endometrium: the inflammatory response to semen. Animal Reproduction Science, 68 273-278. 2001.

WEBER, J.A., WOODS, G.L. Ultrasonographic measurement of stallion accessory sex glands and excurrent ducts during seminal emission and ejaculation. Biology Reproduction, 49, 267273, 1993. 